\title{
Relationship between Masseter Muscle Form and Occlusal Supports of Remaining Teeth
}

\author{
MAKOTO TETSUKA*,**, TSUYOSHI SAGA**, MORIYOSHI NAKAMURA*,**, \\ YOKO TABIRA**, JINGO KUSUKAWA* AND KOH-ICHI YAMAKI** \\ *Dental and Oral Medical Center and ** Department of Anatomy, \\ Kurume University, Kurume 830-0011, Japan
}

Received 7 February 2012, accepted 17 July 2012

\begin{abstract}
Summary: To chew, it is necessary to maintain harmony between the masseter muscle and other organs. Various studies have been conducted on the masseter muscle, but none has examined the relationships among masseter muscle form, occlusal support of remaining teeth, and maxillofacial morphology. Thus, we conducted the present study using cadavers donated to anatomy practice. After the masseter muscle was extracted, its length, width, thickness, and volume were measured; histological observations were conducted; and the muscle fiber cross-sectional area and muscle density were calculated. In addition, denture use and non-use were examined. The results showed that when regional support loss occurs, muscle fiber thickness and density decrease. This in turn causes masseter muscle thickness and volume to decrease, resulting in muscle atrophy. Furthermore, excluding Eichner class A cases (all regions intact), the thickness of the masseter muscle is greatest when the premolar support region remains. The premolar support region was shown to have the most impact on masseter muscle morphology. These results suggest that atrophy of the masseter muscle can be arrested or improved with the use of dentures in the case of tooth loss.
\end{abstract}

Key words masseter muscle form, Eichner classification, remaining teeth, denture.

\section{INTRODUCTION}

To chew effectively, various mouth organs, including the muscles of mastication, the teeth, and the tongue, must work in harmony [1]. Sufficient chewing is related to maintenance of total body health. Therefore, to recover masticatory function, prompt treatment is necessary when it becomes difficult to chew because of problems such as tooth loss [2]. Recently, the development of computed tomography (CT), magnetic resonance imaging (MRI), and ultrasonic devices has made it possible to study the relationships among construction masseter muscle volume, sectional area, and mandible face area in regard to the muscles of mastication [3-6]. The masseter muscle plays a leading role in chewing motion and is located near the surface of the face; therefore, studies on its relationship to jaw form [4,7,8], active muscle mass [9], bite force $[7,8,10-13]$, and muscle changes with aging have been performed [14-19]. The volume of the masseter muscle has predominantly been evaluated in living patients using CT, MRI, and ultrasonic devices [3-6].

Teeth are also important for chewing. However, tooth loss occurs as a result of tooth decay, periodontitis, and injuries. Therefore, it is necessary to note the state of the remaining teeth when treating the defective area. In these cases, the Eichner classification $[20,21]$ is used to determine the state of the remaining teeth. When two or fewer occlusal support regions according to the Eichner classification remain, the remaining regions collapse easily [22]. This is reportedly caused by a disruption in the balance of the right and left articulation and leads to symptoms throughout the body [1].

Corresponding author: Makoto Tetsuka, Department of Anatomy, Kurume University School of Medicine, 67 Asahi-machi, Kurume 830-0011, Japan. Tel: +81-942-31-7540 Fax: +81-942-33-3233 E-mail:1anatomy@med.kurume-u.ac.jp 
Thus, various studies have been conducted concerning the masseter muscle and teeth [3-19]. However, we have found no research on the relationship between the form of the masseter muscle and remaining teeth or between the form of the masseter muscle and maxillofacial morphology using cadavers. In addition, how changes in the condition of the remaining teeth influence the masseter muscle remains unclear. We performed this study to clarify whether the use of dentures benefits the masseter muscle. We measured the length, width, thickness, and volume of actual masseter muscles taken from cadavers. Advantages of this method include the fact that masseter muscles can be fully removed from cadavers, the muscle form can be directly observed and measured, and large-scale machines are unnecessary. In terms of the condition of the remaining teeth, the Eichner classification expresses only the number of support regions; thus, it was impossible to judge whether the support regions existed on the right or left or whether the premolar and/or molar regions remained. In this study, support regions were classified by differences in the number of right and left support regions as well as by premolar and molar regions to further divide the Eichner classification, and the effect of tooth loss on the masseter muscle was considered using both the Eichner classification and our more detailed classification.

\section{MATERIALS AND METHODS}

\section{Materials}

Fifty-six cadavers that had been donated for anatomy study to the Kurume University School of Medicine in 2007 and 2008 were made available for study of the relationship between masseter muscle form and remaining teeth. These cadavers had a mean age of 78.1 years (48-100 years). In 2009, 14 additional cadavers were made available for study of the relationship between the histological features of the masseter muscle and the remaining teeth. These cadavers had a mean age of 74.2 years (68-96 years). The cadavers were injected with 15 to $20 \mathrm{~L}$ of $10 \%$ to $15 \%$ formalin solution via the femoral artery. The cadavers had been fixed for a period of 3 to 12 months.

The protocol for the present research did not require approval by the ethics committees of our institutions, and it conformed to the provisions of the Declaration of Helsinki in 1995 (as revised in Edinburgh 2000).

\section{Methods}

\section{Measurement of masseter muscle}

The skin and hypodermal tissue under the skin

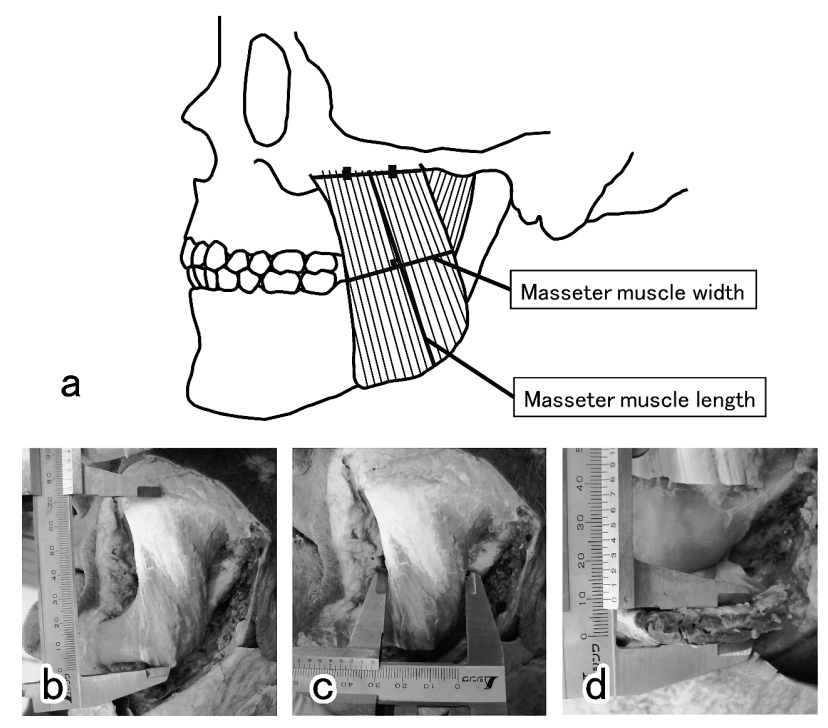

Fig. 1. Measurment of masseter muscle form. a: Schema of the measurement of the masseter muscle width and length. b: The length of the masseter muscle was assumed to be the distance from the center of the origin to the angle of the mandibular bone. c: The width of the muscle was measured by locating the intersection of the upper side of the lower jawbone. d: The masseter muscle was cut, and the thickness of the masseter muscle was measured along the line of the width.

were removed from the masseter muscles and the masseter muscle itself was completely resected. Each part of the left and right masseter muscle length, width, and thickness was measured with calipers (Figs. 1a-d).

\section{Measurement and calculation of volume of masseter muscle}

To formulate a new method to calculate masseter muscle volume, the volume of the masseter muscle was measured using the water displacement method (W.D. method) [23] and the silicon impression material method.

W.D. method: Masseter muscles of nine randomly selected cadavers were first measured (length, width, and thickness) and then extracted. The extracted masseter muscle was submerged into a measuring cylinder containing water, and the volume of the masseter muscles was determined from the water displacement.

Silicon impression method: After measurement by the W.D. method, the silicon was kneaded to eliminate air from the impression material (GC, Eguzafain, Regular type; GC, Tokyo), and the masseter muscle was pressed into the silicon. After the impression material hardened, the masseter muscle was removed. The space that had contained the masseter muscle was filled with water, and the volume of the water was measured. 
Proposed formula for masseter muscle volume: The masseter muscle was thought of as a rectangular parallelepiped (a rectangular cube), therefore the formula used to calculate the volume was: volume of masseter muscle $=$ length $\times$ width $\times$ thickness. The volume of the masseter muscle from nine cadavers was calculated using this formula. We compared the results of W.D. method, Silicon impression method, and Formula for masseter muscle volume.

\section{Confirmation and classification of remaining} teeth and relationship between condition of remaining teeth and the length, width, thickness, and volume of the masseter muscle

The number of teeth was investigated following the Eichner classification [20,21] (Table 1). When considering Eichner class B, subjects with partial dentures and those without dentures were noted. We classified the occlusal support region according to the Eichner classification in terms of the number of occlusal support regions, taking into account the use or non-use of partial dentures, and examined the relationships with the masseter muscle form. In the case of partial dentures, the occlusal support region comprised a natural tooth and denture and one natural tooth together with a partial denture together were counted as one occlusal support region. There were no occlusal support regions in Eichner class $\mathrm{C} 1$ or $\mathrm{C} 2$, however, when considering class $\mathrm{C} 3$, denture users and denture non-users were noted. During prosthodontic treatment, teeth with caries three and caries four are extracted before treatment. Therefore, these teeth were not counted as support regions according to the Eichner classification and were not included in the number of support regions in this study. Moreover, the Eichner classifica-

TABLE 1.

Classification of Eichner of remaining teeth [20] modified from Ikebe et al. [21]

A : Group A have occlusal contacts in all four posterior support zones.

A-1 : No missing teeth in the mandible and maxilla.

A-2 : At least one missing tooth in either the mandible or maxilla.

A-3 : At least one missing tooth in both the mandible and maxilla.

B : Group B have occlusal contacts in three to one zone(s) of contact or in the anterior region only.

B-1 : Posterior occlusal contacts in three zones.

B-2 : Posterior occlusal contacts in two zones.

B-3 : Posterior occlusal contact in one zone.

B-4 : Occlusal contact(s) in the anterior region only.

$\mathrm{C}$ : Group $\mathrm{C}$ have no occlusal contact at all.

C-1 : At least one tooth in both the mandible and maxilla without any occlusal contact.

C-2 : At least one tooth in either the mandible or maxilla.

C-3 : Full edentulous in both arches. Denture or

Non Denture

TABLE 2.

Classification method according to differences in number of right and left support regions

(Classification (1)) and classification according to premolar and molar support regions

(Classification (2)) (The class B of the classification of Eichner was subdivided)

\begin{tabular}{|c|c|c|c|c|}
\hline $\begin{array}{l}\text { Number of right and left } \\
\text { support region }\end{array}$ & $\begin{array}{l}\text { Right } \\
\text { support } \\
\text { region }\end{array}$ & $\begin{array}{l}\text { Left } \\
\text { support } \\
\text { region }\end{array}$ & $\begin{array}{c}\text { Classification } \\
\text { (1) }\end{array}$ & $\begin{array}{c}\text { Classification } \\
\text { (2) }\end{array}$ \\
\hline B-1 & 1 & 2 & B-1-R1L2 & \multirow[t]{2}{*}{ B-b } \\
\hline 3 support regions & 2 & 1 & B-1-R2L1 & \\
\hline \multirow{3}{*}{$\begin{array}{c}\text { B-2 } \\
2 \text { support regions }\end{array}$} & 0 & 2 & \multirow{3}{*}{$\begin{array}{l}\text { B-2-L2 } \\
\text { B-2-R1L1 } \\
\text { B-2-R2 }\end{array}$} & B-a \\
\hline & 1 & 1 & & B-b \\
\hline & 2 & 0 & & B-c \\
\hline \multirow{2}{*}{$\begin{array}{c}\text { B-3 } \\
1 \text { support region }\end{array}$} & \multirow{2}{*}{$\begin{array}{l}0 \\
1\end{array}$} & \multirow{2}{*}{$\begin{array}{l}1 \\
0\end{array}$} & \multirow{2}{*}{$\begin{array}{l}\text { B-3-L1 } \\
\text { B-3-R1 }\end{array}$} & B-a \\
\hline & & & & $\begin{array}{l}\text { B-b } \\
\text { B-c }\end{array}$ \\
\hline $\begin{array}{c}\text { B-4 } \\
0 \text { support regions }\end{array}$ & 0 & 0 & B-4-R0L0 & B-d \\
\hline
\end{tabular}

Classification (2) B-a : Only the premolar region remains as a support region

$\mathrm{B}-\mathrm{b}$ : The support region is not greater than three, and the molar region and the premolar region remain

B-c : Only the molar region remains as a support region

$\mathrm{B}-\mathrm{d}$ : There is no remaining support region 
tion considers only the total number of support regions. This classification is inadequate to compare the relationship between right and left and between premolar and molar regions to masseter muscle form. Therefore, when observing Eichner class B, we subdivided the traditional classification to clarify these additional factors (Table 2).

\section{Examination by light microscopy}

For each of the 6 types of cases from 4 support regions to 1 support region according to the Eichner classification as well as cases with no remaining teeth both with and without full dentures, masseter muscles from 14 cadavers were extracted and re-fixed in $10 \%$ formalin. Paraffin sections $5 \mu \mathrm{m}$ in thickness and perpendicular to the muscle fiber were then prepared as usual and observed after hematoxylin and eosin staining.

5. Measurement of cross-sectional area of one muscle fiber, number of muscle fibers, muscle fiber density, area density of muscle fiber, and tissue space among the muscle fibers

After observing and photographing the prepared specimens using light microscopy, the thickness of the muscle fibers of each masseter muscle was compared. Using area measurement software, the cross-sectional area of one muscle fiber from each specimen was measured and compared. This was necessary because the muscle fiber is not a perfect circle. In addition, to determine muscle fiber density, the number of muscle fibers per $1 \mathrm{~mm}^{2}$ was counted. From these results, area density and tissue space were calculated as follows: Area density $(\%)=$ Average area of one muscle fiber $\times$ Number of muscle fibers in $1 \mathrm{~mm}^{2} \div \mathrm{mm}^{2} \times 100$; tissue space $=$ unit area $1 \mathrm{~mm}^{2}$ (Number of muscle fib- ers $\times$ Average area of one muscle fiber). Calculations were performed to correlate the number of occlusal support regions and tissue space.

6. The correlations between the number of teeth and the length, width, thickness and volume of the masseter muscle

\section{RESULTS}

\section{Results of measurement of the masseter muscle}

The average length of the masseter muscle among 56 cadavers was $65.7 \pm 7.1 \mathrm{~mm}$. The average length of the right and left masseter muscles was $65.9 \pm 7.3 \mathrm{~mm}$, and $65.6 \pm 7.2 \mathrm{~mm}$, respectively.

The verage width of the masseter muscle among 56 cadavers was $35.2 \pm 3.5 \mathrm{~mm}$. The average width of the right and left masseter muscles was $35.2 \pm 3.8 \mathrm{~mm}$, and $35.2 \pm 3.9 \mathrm{~mm}$, respectively.

The average thickness of the masseter muscle among 56 cadavers was $6.5 \pm 2.5 \mathrm{~mm}$. The average thickness of the right and left masseter muscles was $6.7 \pm 2.6 \mathrm{~mm}$, and $6.4 \pm 2.5 \mathrm{~mm}$, respectively.

\section{Masseter muscle volume}

By the W.D. method, the average volume of the right masseter muscle was $22.1 \mathrm{~cm}^{3}$ and that of the left masseter muscle was $21.4 \mathrm{~cm}^{3}$.

By the silicon impression method, the average of volume of the right masseter muscle was $21.8 \mathrm{~cm}^{3}$ and that of the left masseter muscle was $21.1 \mathrm{~cm}^{3}$.

By our proposed formula, the average of volume of the right masseter muscle was $21.3 \mathrm{~cm}^{3}$ and that of the left masseter muscle was $20.7 \mathrm{~cm}^{3}$.

TABLE 3.

Measured values of the masseter muscle form

\begin{tabular}{|c|c|c|c|c|c|c|c|c|c|c|}
\hline & & \multirow{2}{*}{$\mathrm{n}$} & \multicolumn{2}{|c|}{ Length (mm) } & \multicolumn{2}{|c|}{ Width $(\mathrm{mm})$} & \multicolumn{2}{|c|}{ Thickness (mm) } & \multicolumn{2}{|c|}{ Volume $\left(\mathrm{cm}^{3}\right)$} \\
\hline & & & Right & Left & Right & Left & Right & Left & Right & Left \\
\hline A-1 & & 1 & 72.7 & 69.0 & 36.0 & 36.0 & 10.0 & 7.0 & 26.2 & 17.4 \\
\hline A-2 & & 1 & 78.0 & 76.5 & 42.0 & 42.0 & 8.0 & 5.0 & 26.2 & 16.1 \\
\hline A-3 & & 1 & 72.9 & 74.7 & 40.0 & 40.4 & 8.2 & 8.9 & 23.9 & 26.9 \\
\hline B-1 & & 3 & $67.1 \pm 10.9$ & $65.6 \pm 9.7$ & $35.5 \pm 6.9$ & $35.5 \pm 7.7$ & $11.2 \pm 1.4$ & $10.0 \pm 1.6$ & $27.5 \pm 10.8$ & $24.0 \pm 10.5$ \\
\hline B-2 & & 3 & $62.2 \pm 7.2$ & $65.2 \pm 7.3$ & $37.5 \pm 4.9$ & $38.2 \pm 4.2$ & $9.3 \pm 6.1$ & $9.0 \pm 5.6$ & $23.7 \pm 18.3$ & $24.5 \pm 19.3$ \\
\hline B-3 & & 6 & $69.5 \pm 6.3$ & $68.5 \pm 6.8$ & $37.5 \pm 1.9$ & $37.3 \pm 1.2$ & $6.6 \pm 2.5$ & $6.2 \pm 2.1$ & $17.4 \pm 7.0$ & $15.8 \pm 5.9$ \\
\hline B-4 & & 3 & $66.0 \pm 14.2$ & $63.0 \pm 14.3$ & $35.5 \pm 4.3$ & $37.8 \pm 1.6$ & $7.6 \pm 3.2$ & $6.5 \pm 3.1$ & $19.3 \pm 13.8$ & $16.6 \pm 11.1$ \\
\hline C-1 & & 8 & $67.4 \pm 4.8$ & $65.8 \pm 4.4$ & $36.8 \pm 3.3$ & $36.0 \pm 2.2$ & $6.7 \pm 1.4$ & $6.7 \pm 1.6$ & $16.5 \pm 4.0$ & $15.9 \pm 4.3$ \\
\hline $\mathrm{C}-2$ & & 6 & $65.4 \pm 7.8$ & $68.7 \pm 8.1$ & $33.7 \pm 4.6$ & $35.1 \pm 3.6$ & $5.2 \pm 2.1$ & $4.8 \pm 1.5$ & $12.2 \pm 7.6$ & $11.7 \pm 5.3$ \\
\hline \multirow{2}{*}{$\mathrm{C}-3$} & {$\left[\begin{array}{l}\text { Non } \\
\text { Denture }\end{array}\right.$} & 12 & $64.6 \pm 7.8$ & $63.5 \pm 5.0$ & $33.6 \pm 3.1$ & $32.7 \pm 3.4$ & $5.2 \pm 1.3$ & $4.9 \pm 1.4$ & $11.5 \pm 4.1$ & $10.5 \pm 3.8$ \\
\hline & $\mathrm{L}_{\text {Denture }}$ & 12 & $62.9 \pm 7.7$ & $63.5 \pm 8.3$ & $33.5 \pm 2.4$ & $33.6 \pm 4.0$ & $6.6 \pm 2.4$ & $6.7 \pm 2.5$ & $14.2 \pm 6.0$ & $14.7 \pm 7.5$ \\
\hline
\end{tabular}


These calculated results were in agreement with those obtained by both the W.D. method and the silicon impression method. Therefore, the volume of the masseter muscle of the other 47 cadavers was obtained by this formula.

\section{Relationship between condition of remaining teeth and the length, width, thickness, and volume of the masseter muscle}

3-1) Measured values of the masseter muscle form in Eichner classification (Table 3)

Concerning muscle length in class $\mathrm{B}$, no significant correlation $(\mathrm{r}=-0.026)$ was found between the number of occlusal support regions and the length of the masseter muscle (Fig. 2a). Concerning the width of class B, no significant correlation $(r=-0.099)$ was found between the number of occlusal support regions and the width of the masseter muscle (Fig. 2b). Concerning the thickness of class $\mathrm{B}$, a significant correlation $(\mathrm{r}=0.449 ; \mathrm{p}<0.01)$ was noted between the number of occlusal support regions and the thickness of the masseter muscle (Fig. 2c).

In addition, in cases where partial dentures were not used and only natural teeth were counted as oc- clusal support regions, no significant relationship was found between occlusal support region and thickness or volume of the masseter muscle (Figs. 3a, b). The number of occlusal support regions increased with use of a partial denture. In cases with a partial denture, we also examined the Eichner classification and the relationships between the number of occlusal support regions and masseter muscle form (thickness and volume). We found that as the number of occlusal support regions decreased from class B1 to B4, the masseter muscle thickness and volume also decreased (Figs. 3c, d). In addition, the masseter muscles of partial denture wearers in class B were thicker than those of non-denture wearers. In class $\mathrm{C}$ on both the right and the left, the thickness of the masseter muscle decreased from $\mathrm{C} 1$ to $\mathrm{C} 2$, but the muscle in $\mathrm{C} 3$ was thicker than that in $\mathrm{C} 2$. In addition, a comparison was made between full denture wearers and non-denture wearers in class C3 with upper and lower edentulous jaws. The masseter muscles of the full denture wearers were thicker than those of non-denture wearers.

With regard to muscle volume in class $\mathrm{B}$, a significant correlation $(r=0.320 ; p<0.05)$ was observed between the number of occlusal support regions and the

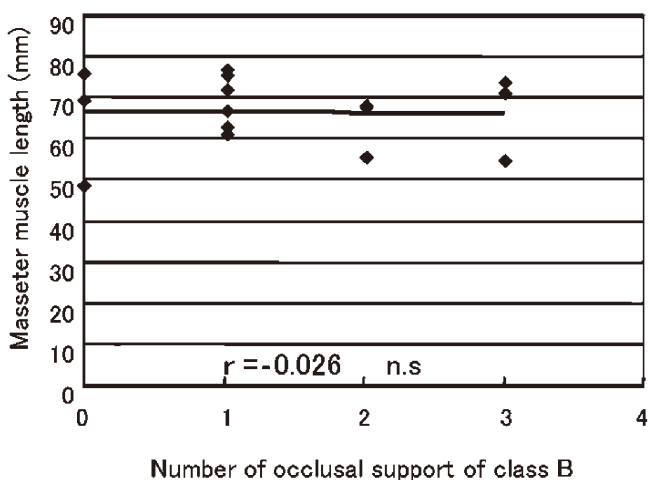

C

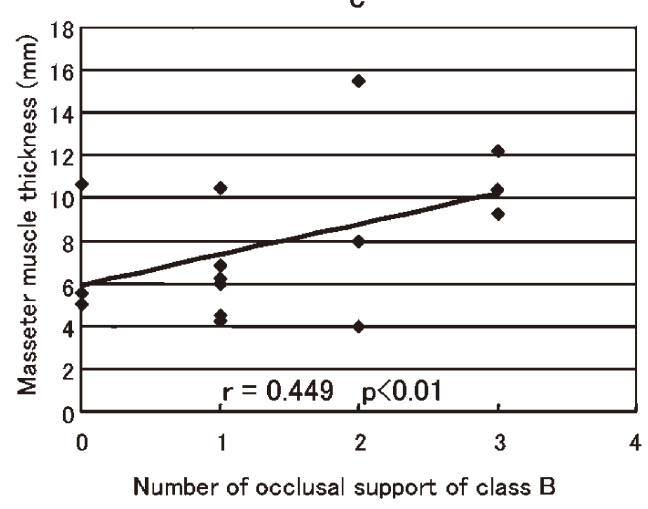

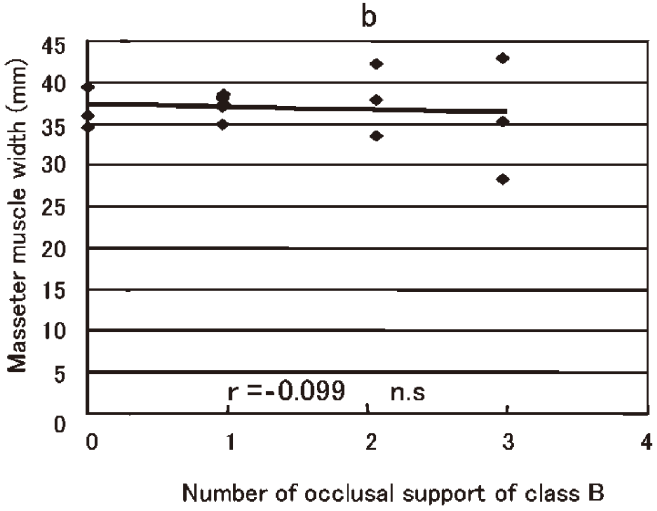

d

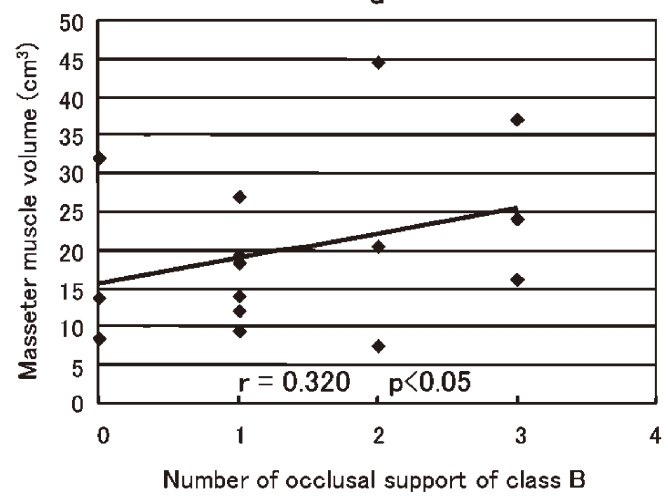

Fig. 2. Relationship between the classification of Eichner and masseter muscle form. a: length, b: width, c: thickness, d: volume. 

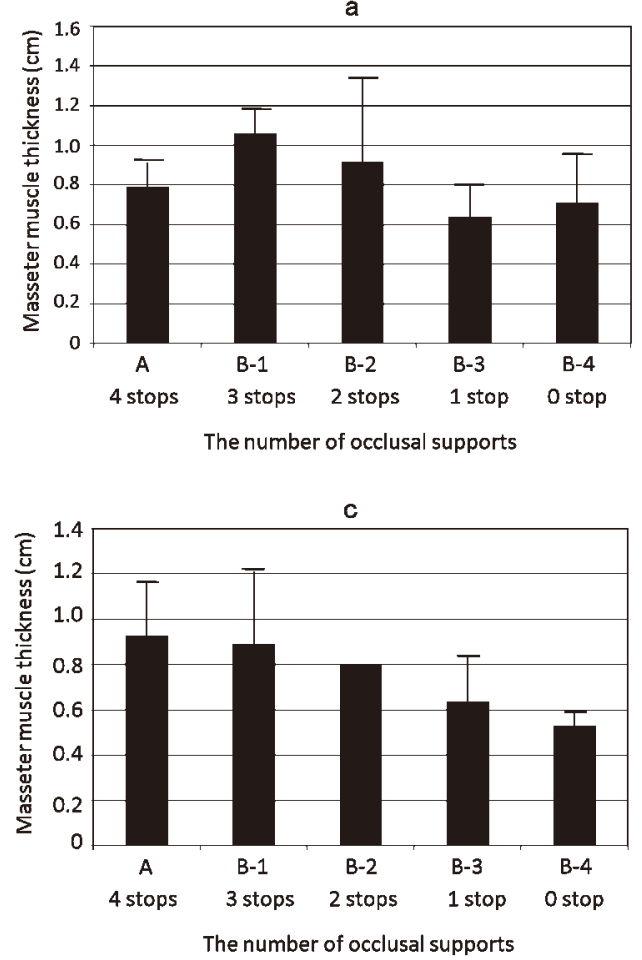

b

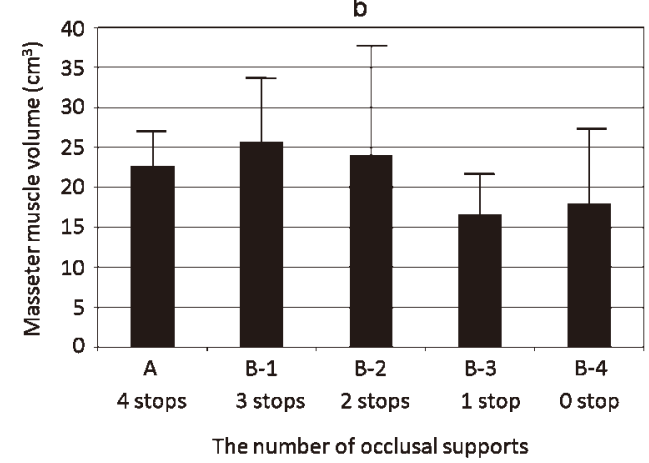

d

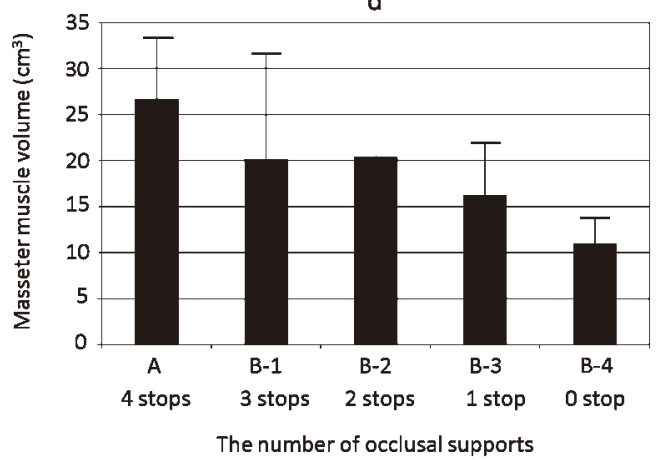

Fig. 3. Relationship between the masseter muscle form and the number of occlusal support regions of natural tooth and partial denture.

$\mathrm{a}, \mathrm{b}$ : In the case of class B including the partial denture use or non-use, occlusal support region made only natural tooth. a: Relationship between occlusal support region of natural tooth and thickness of the masseter muscle. b: Relationship between occlusal support region of natural tooth and volume of the masseter muscle.

$\mathrm{c}, \mathrm{d}$ : In the case of class B including the partial denture use or non-use, occlusal support region made natural tooth and partial denture. c: Relationship between occlusal support region of natural tooth and partial denture and the thickness of the masseter muscle. d: Relationship between occlusal support region of natural tooth and partial denture and the volume of the masseter muscle.

volume of the masseter muscle (Fig. 2d). In addition, the volume of the masseter muscle decreased from B1 to B4 as the number of occlusal support regions decreased. In class $\mathrm{C}$, the volume of the masseter muscle decreased from $\mathrm{C} 1$ to $\mathrm{C} 2$, but increased in $\mathrm{C} 3$. In addition, the volume of the masseter muscle of full denture wearers was larger than that of non-denture wearers.

3-2) Relationship between the difference in number of occlusal support regions and masseter muscle form

As previously mentioned, the relationship between the state of the remaining support regions on the right and left and the masseter muscle was investigated in Eichner class B. We further divided Eichner class B into B1 (B1-R1L2, B1-R2L1), B2 (B2-L2, B2-R1L1, B2-R2), and B3 (B3-L1, B3-R1) and compared the difference in the number of occlusal support regions and masseter muscle form (Table 2). We used dual independent sampling tests for each combination of re- maining right and left teeth. No significant differences were observed between the right and left with regard to number of occlusal support regions and masseter muscle form.

3-3) Relationship between the condition of remaining occlusal premolar and molar support regions for Eichner class $B$ and masseter muscle form

The length, thickness, and volume of the masseter muscle, but not the width, were greatest for B-a combinations and smallest for B-c combinations (Table $4)$.

\section{Optical microscopic observation}

The right and left masseter muscles from 14 cadavers that had been categorized according to the Eichner classification were histologically observed (Fig. 4) [class A, n=3, Fig. 4a; class B (B1), n=1, Fig. 4b; class B (B2), n=3, Fig. 4c; class B (B3), n=1, Fig. 4d; class 
$\mathrm{C}(\mathrm{C} 3$, non-denture wearers), n=3, Fig. 4e; and class $\mathrm{C}$ (C3, denture wearers), $n=3$, Fig. 4f]. The average crosssectional area of each muscle fiber, the number of muscle fibers per unit area (density), and the area density of muscle fibers were calculated (Table 5).

\section{Lateral cross-sectional area of an average masseter muscle fiber and the number of muscle fibers per unit area for each group}

A dual independent sampling was conducted to obtain an average cross-sectional area per muscle fiber of the masseter muscle. There was a significant difference between right and left for classes B1, B3, and edentulous jaws (C3) with no dentures, but no such difference was observed for classes A, B2, and edentulous jaws (C3) with dentures. In addition, there was no significant correlation between the number of occlusal support regions and the cross-sectional area of a masseter muscle fiber $(\mathrm{r}=0.511)$.

In the same way, the number of muscle fibers per

TABLE 4.

Relationship between the condition of remaining occlusal premolar and molar support regions for Eichner's class B and masseter muscle form

\begin{tabular}{lccccc}
\hline Occlusal supports & $\mathrm{N}$ & $\begin{array}{c}\text { Length } \\
(\mathrm{mm})\end{array}$ & $\begin{array}{c}\text { Width } \\
(\mathrm{mm})\end{array}$ & $\begin{array}{c}\text { Thickness } \\
(\mathrm{mm})\end{array}$ & $\begin{array}{c}\text { Volume } \\
\left(\mathrm{cm}^{3}\right)\end{array}$ \\
\hline $\begin{array}{l}\text { Premolar teeth } \\
\text { (B-a) }\end{array}$ & 7 & $68.5 \pm 6.2$ & $37.8 \pm 4.2$ & $9.1 \pm 3.8$ & $24.1 \pm 11.8$ \\
\hline $\begin{array}{l}\text { Premolar and Molar teeth } \\
\text { (Less than 3 steps) } \\
\text { (B-b) }\end{array}$ & 4 & $64.1 \pm 9.7$ & $35.1 \pm 5.6$ & $9.0 \pm 3.8$ & $21.3 \pm 12.9$ \\
\hline $\begin{array}{l}\text { Molar teeth } \\
\text { (B-c) }\end{array}$ & 1 & 62.0 & 38.5 & 6.0 & 14.3 \\
\hline $\begin{array}{l}\text { No occlusal supports } \\
\text { (B-d) }\end{array}$ & 3 & $66.8 \pm 8.3$ & $36.7 \pm 2.2$ & $6.2 \pm 2.5$ & $15.8 \pm 7.6$ \\
\hline
\end{tabular}
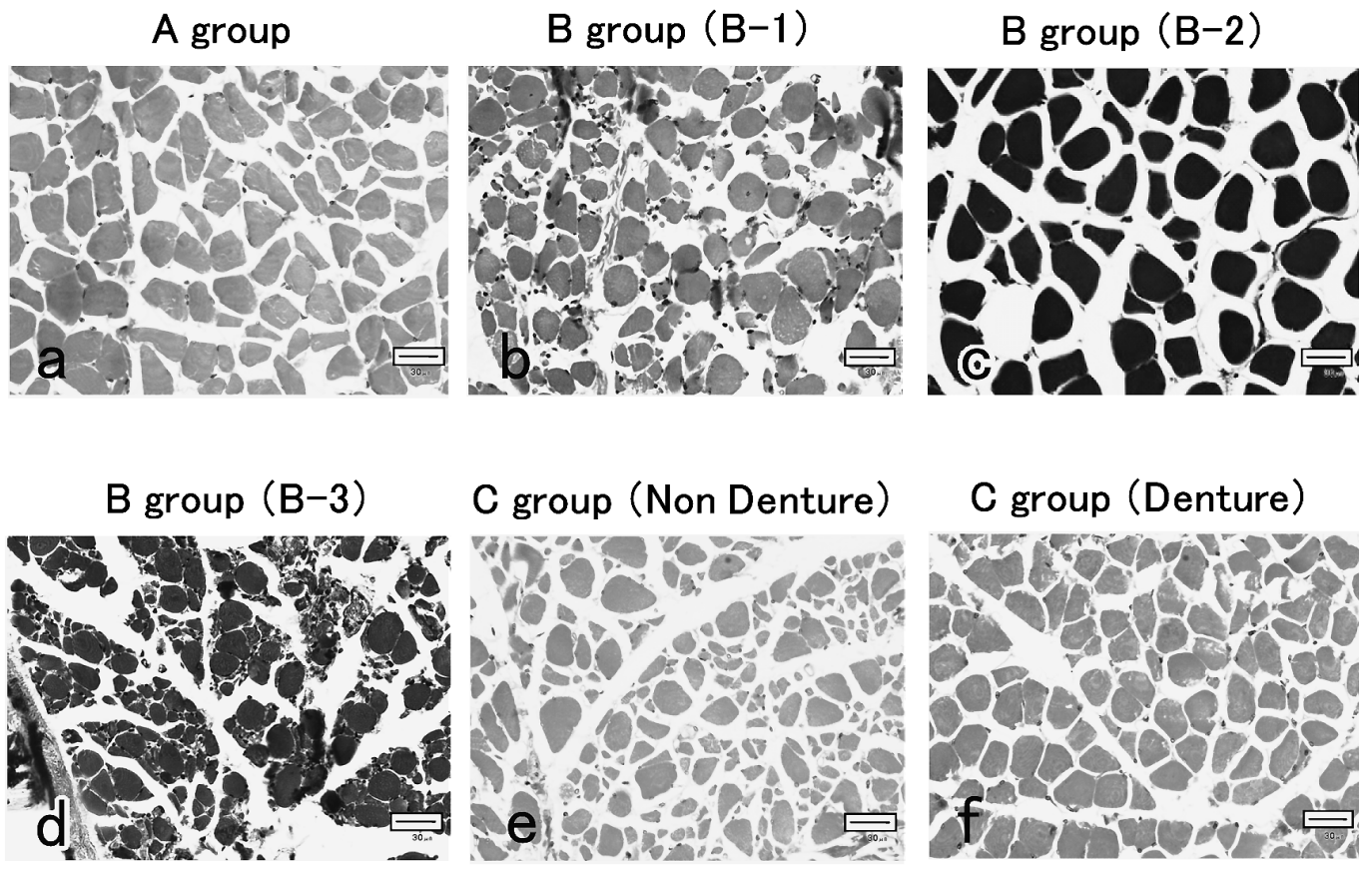

Fig. 4. A histological observation of the right and left masseter muscle that had been categorized according to the classification of Eichner. (H\&E staining of masseter muscle) a: Eichner's class A (4 stops). b: Eichner's class B (B-1) (3 stops). c: Eichner's class B (B-2) (2 stops). d: Eichner's class B (B-3) (1 stop). e: Eichner's class C (0 stop; No denture). f: Eichner's class C (0 stop; Denture usage). Scale bars $=30 \mu \mathrm{m}$ 
TABLE 5.

We had been categorized according to the Eichner classification were histologically observed and the average cross-sectional area of each muscle fiber, the number of muscle fibers per unit area (density), and the area density of muscle fibers were calculated

\begin{tabular}{|c|c|c|c|}
\hline & & Right & Left \\
\hline \multirow{3}{*}{ Class A } & Closs-sectional $\left(\mu \mathrm{m}^{2}\right)$ & $747.7 \pm 179.7$ & $686.9 \pm 176.8$ \\
\hline & Density (fuber/mm²) & $839.8 \pm 90.4$ & $1175.1 \pm 116.6$ \\
\hline & Area density (\%) & $62.8 \pm 1.6$ & $80.7 \pm 2.1$ \\
\hline \multirow{3}{*}{$\begin{array}{c}\text { Class B } \\
\text { B-1 }\end{array}$} & Closs-sectional area $\left(\mu \mathrm{m}^{2}\right)$ & $365.3 \pm 184.5$ & $499.9 \pm 160.9$ \\
\hline & Density (fuber/mm²) & $1697.1 \pm 107.9$ & $1111.0 \pm 84.6$ \\
\hline & Area density (\%) & $62.0 \pm 2.0$ & $55.5 \pm 1.4$ \\
\hline \multirow{3}{*}{$\begin{array}{c}\text { Class B } \\
\text { B-2 }\end{array}$} & Closs-sectional area $\left(\mu \mathrm{m}^{2}\right)$ & $832.8 \pm 255.8$ & $745.2 \pm 183.5$ \\
\hline & Density (fuber/mm²) & $632.8 \pm 70,0$ & $656.1 \pm 64.2$ \\
\hline & Area density (\%) & $52.7 \pm 1.8$ & $48.9 \pm 1.2$ \\
\hline \multirow{3}{*}{$\begin{array}{c}\text { Class B } \\
\text { B-3 }\end{array}$} & Closs-sectional area $\left(\mu \mathrm{m}^{2}\right)$ & $359.0 \pm 121.9$ & $200.5 \pm 34.2$ \\
\hline & Density (fuber/mm²) & $2338.6 \pm 452.0$ & $4269.0 \pm 577.4$ \\
\hline & Area density (\%) & $84.0 \pm 5.5$ & $85.6 \pm 2.0$ \\
\hline Class $\mathrm{C}$ & Closs-sectional area $\left(\mu \mathrm{m}^{2}\right)$ & $339.5 \pm 187.0$ & $479.2 \pm 164.8$ \\
\hline $\mathrm{C}-3$ & Density (fuber $/ \mathrm{mm}^{2}$ ) & $2834.4 \pm 271.2$ & $1119.7 \pm 119.6$ \\
\hline Non denture & Area density (\%) & $96.2 \pm 5.1$ & $53.7 \pm 2.0$ \\
\hline Class C & Closs-sectional area $\left(\mu \mathrm{m}^{2}\right)$ & $638.2 \pm 138.1$ & $614.6 \pm 143.3$ \\
\hline $\mathrm{C}-3$ & Density (fuber/mm²) & $1338.4 \pm 87.5$ & $1417.2 \pm 105.0$ \\
\hline denture & Area density $(\%)$ & $85.4 \pm 1.2$ & $87.1 \pm 1.5$ \\
\hline
\end{tabular}

unit area was examined. For classes A, B1, B3, and edentulous jaws (C3) with no denture usage, there was a significant difference between right and left, but for B2 and edentulous jaws (C3) with no dentures, no such difference was observed. In addition, no significant correlation was found between the number of occlusal support regions and the number of muscle fibers $(\mathrm{r}=-0.668)$.

We examined whether there was a correlation between the average cross-sectional area of one masseter muscle fiber and the number of muscle fibers per unit area. The number of muscle fibers per unit area increased when the average cross-sectional area of one masseter muscle fiber was small; on the other hand, the number of muscle fibers per unit area decreased when the average cross-sectional area of one masseter muscle fiber was large (negative correlation $[\mathrm{r}=-0.874$, $\mathrm{p}<0.01]$ ) (Fig. 5).

As regards the relation between the number of occlusal support regions and tissue space, tissue space tended to increase as the number of occlusal support regions decreased. However, the correlation was not significant $(\mathrm{r}=0.431)$.

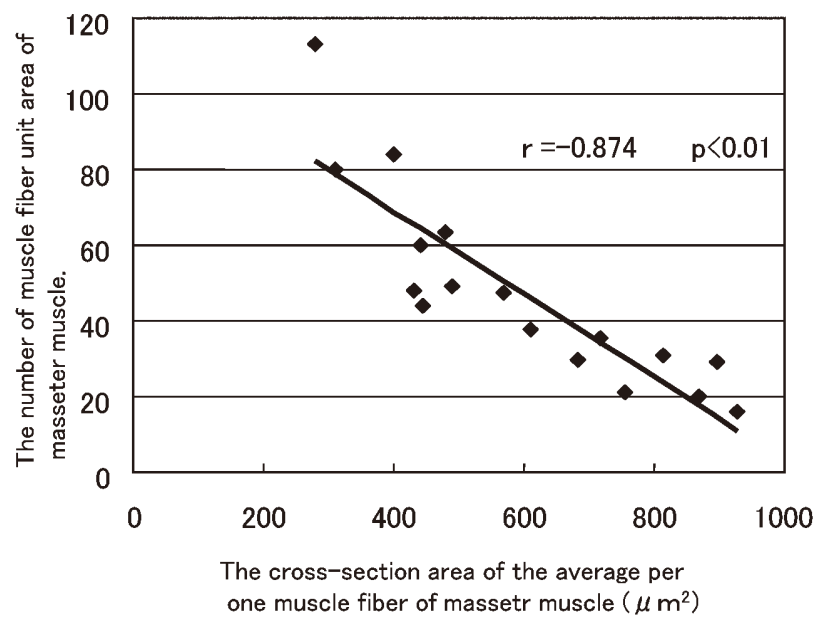

Fig. 5. Relationship between the average area of a crosssection of one masseter muscle fiber and the number of muscle fibers per unit area.

\section{Relationship between the number of teeth and mas- seter muscle form (Figs. 6a-d)}

We determined the correlation between the number of teeth and masseter muscle thickness, width, length, 
a

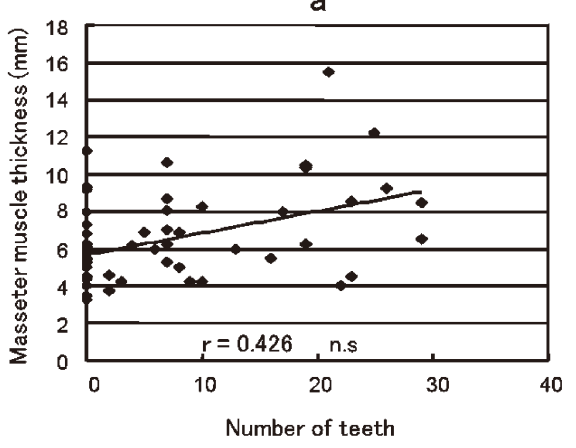

C

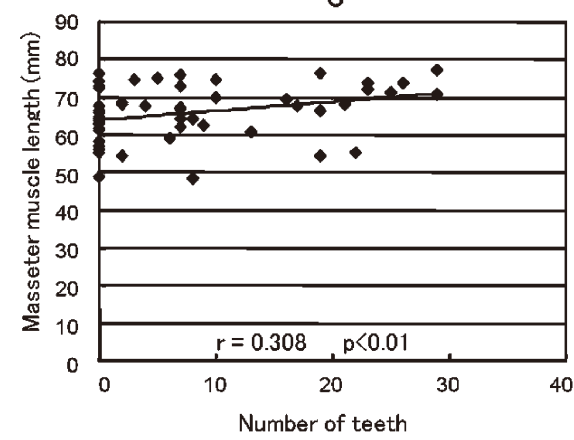

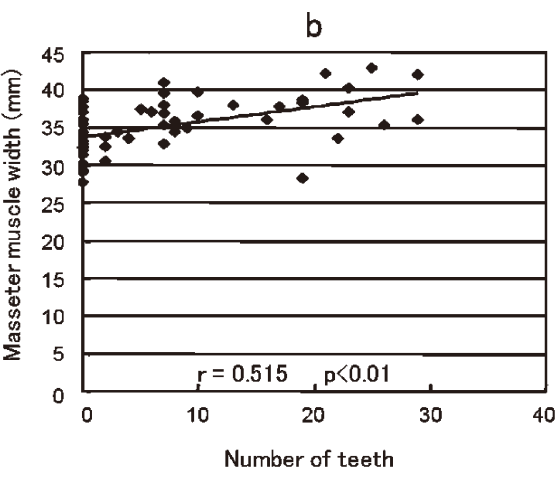

d

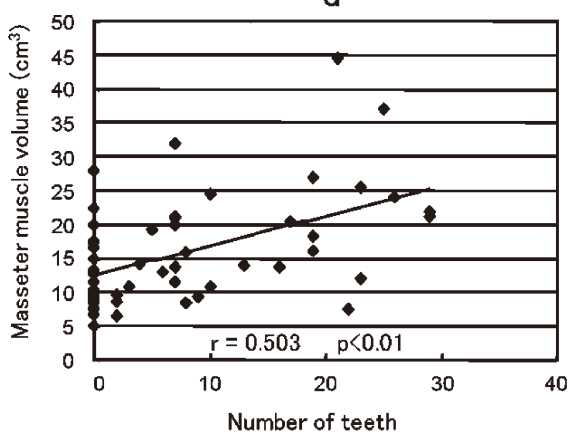

Fig. 6. Relationships between masseter muscle form and number of occlusal support. a: thickness, b: width, c: length, d: volume.

and volume. There was a significant positive correlation between the number of teeth and thickness, width, volume, and length of the masseter muscle.

\section{DISCUSSION}

Several muscles are involved in the chewing process, and these muscles must work in a cooperative, coordinated manner to achieve a balanced chewing movement. The masseter muscle plays an especially principal role in chewing movement and is located in a shallow facial layer; thus, it has been the subject of a variety of studies [3-19]. However, most of these studies were conducted on live subjects rather than cadavers and were thus limited in detail and scope. Therefore, we used cadavers to clarify the relationship between masseter muscle form and remaining teeth and between the masseter muscle and maxillofacial morphology.

Because the volume of the masseter muscle $=$ length $X$ width $\times$ thickness, it was shown that thickness had a strong influence on the masseter muscle volume. We considered thickness from various aspects. In terms of the relationship between the occlusal support region and the thickness of the masseter muscle, we considered that if there are many support regions, mandibular position is stable, but mandibular position becomes unstable when the number of support regions decreases. As a result, muscle function decreases and atrophy occurs. The relationship between occlusal support regions and masseter muscle activity level was studied by Ogasawara [9]. He reported that as the number of occlusal support regions decreased from four to one, the general amount of muscle activity of four muscles decreased as well. Our study showed that a decrease in the number of support regions contributes to a decrease in masseter muscle volume. It is thought that this decrease results in muscle atrophy as the active mass of the muscle decreases. Our results showed that not only the natural teeth but also shared partial dentures functioned as occlusal support regions in class $\mathrm{B}$, similar to the natural dental articulation. Therefore, we were able to confirm the importance of early treatment with both partial and complete dentures.

We believe that individual tendencies to chew on one side or the other influence the relationship between the right and left residual support regions and thickness of the masseter muscle. Kitahara [24] interviewed 
52 men and women regarding which side they tended to chew on and then examined the relationship between the right and left masseter muscle and the dominant chewing side. There was a high correlation between a greater masseter muscle cross-sectional area and the dominant chewing side [24].

In terms of the relationship between the remaining premolar and molar support regions and masseter muscle form, we believe that the premolar support region has great importance and that there is a strong relationship between the masseter muscle and the premolar region.

In terms of the relationship between the denture users and denture non-users and the thickness of the masseter muscle, we consider that if prosthetic measures are taken with the use of dentures when the number of support regions decreases chewing is enabled, mandibular position is stabilized, muscle function is restored, and atrophy can be reversed. This is believed to be why the masseter muscle was thicker in denture users.

In addition, in terms of the masseter muscle of full denture users, various studies have been performed on thickness and biting force, electromyogram results, and the fit of full dentures and their effect on biting force [25]. Watanabe et al. [25] stated that the quality of the dentures used has an effect on the decline in the form and function of masticatory muscles. They reported that wearing appropriate dentures is important for the maintenance of these muscles. In this study, we were able to examine denture use and non-use and their effects on masseter muscle form using cadavers. Although were unable to judge whether the fit of the denture was appropriate, it was shown that the use of dentures had been beneficial.

We observed optical microscopic images of the masseter muscle using hematoxylin and eosin staining for each Eichner class to perform histological examinations and obtain the cross-sectional area of representative muscle fibers and density per square unit. Ihara [26] measured cross sections of 20 masseter muscles from cadavers. The maximum result was 992 fibers $/ \mathrm{mm}^{2}$, the minimum was 380 fibers $/ \mathrm{mm}^{2}$, and the average of all cases was 656 fibers $/ \mathrm{mm}^{2}$. The maximum result was 2.5 times the minimum. In the present study, both the left and right sides of 14 sets of masseter muscles were observed ( 28 cases). The maximum result was 4269 fibers $/ \mathrm{mm}^{2}$, the minimum was 417 fib$\mathrm{ers} / \mathrm{mm}^{2}$, and the average of all cases was 1409 fibers/ $\mathrm{mm}^{2}$. The maximum result was $>10$ times the minimum. We compared our results with those in Ihara's report and considered the cause of our widely varied results. It is thought that this increase in both the maximum and average results may have been caused by the fact that the subjects in this study were younger and had little tooth loss and relatively healthy teeth. In addition, no significant relationships were observed between the number of support regions and muscle cross-sectional area and between the number of support regions and area density.

Even in cadavers, it is evident that as a consequence of tooth loss, support regions decrease and mandibular position becomes unstable. As a result, chewing becomes impossible, meals are necessarily soft, and it is thought that muscle atrophy occurs because myofunction decreases. Underdevelopment of masseter muscle fibers was observed after giving powder and liquid food to mice [27]. A similar change is thought to have occurred in the cadavers' muscle fibers. In fact, muscle fiber restitution had occurred in denture users, but not in non-denture users. Therefore, in non-denture users, the muscle fibers remained thin, whereas muscle fibers were larger in denture users than in non-users. It is thought that with appropriate prosthetic treatment such as dentures or bridges, muscle fibers recover because chewing is enabled. Furthermore, Yamaguchi et al. [28] reported that prevention of and recovery from disuse atrophy of muscles such as the soleus seems to be more effective when exercise is started early. From these reports, it is thought that early prosthetic treatment for tooth loss to maintain proper occlusion and chewing may be connected to prevention of and recovery from masseter muscle atrophy. Atrophy of the muscle fiber occurs with a decrease in biting force when dentures are not used. However, biting force is maintained when dentures are used, and it is supposed that atrophy of the muscle fiber is prevented. The results showed that masseter muscle thickness and volume decrease as the number of support regions from the remaining teeth decreases. This clearly shows the need to maintain support regions and the benefit of dental treatment for elderly and/or handicapped persons [2]. Therefore, the importance of early treatment for odontopathy to prevent tooth loss and prosthetic measures for tooth loss are evident.

We studied the relationship between occlusal support and masseter muscle form. The loss of occlusal support resulted in masseter muscle atrophy. However, appropriate prosthetic treatment to enable sufficient chewing can prevent this atrophy and serve to maintain or recover the function of the masseter muscle. The findings of this study show a close relationship between occlusal support and masseter muscle form. 


\section{CONCLUSION}

The thickness and volume of the masseter muscle decreased with a decrease in the number of supporting regions of the remaining teeth. This relationship was especially pronounced when observing the condition of the premolar region. Denture use substantially improved atrophy of the masseter muscle in subjects with edentulous jaws. This finding emphasizes the benefits of denture use.

\section{REFERENCES}

1. Muratsu K, Fujino T, Kikkawa K, and Horie J. Study of lively elderly from dental aspects (2). J Health Sci 1992; 14:43-47.

2. Suzuki M, Saitou E, Oguchi K, and Katou T. Dental care of an old handicapped person and about an effect for the obstacle. Japan Dental Association Journal 1999; 52:608617.

3. Weijs WA, and Hillen B. The relation ship between the physiological cross-section of the human jaw muscles and their cross-sectional area in computer tomograms. Acta anat. 1984; 118:129-138.

4. Nagaki E, Yotsui Y, and Kawamoto T. Relationship between the maxillofacial morphology and the masseter muscle. Orthod Waves-Jpn Ed 2004; 63:1-10.

5. Higashi K, Goto T, Kanda S, Horinouchi Y, Shiratsuchi Y et al. A morphological study of the masseter muscle using magnetic resonance imaging in patients with jaw deformities. Cases demonstrating mandibular deviation. J Jpan Stomatol Soc 2006; 55:17-22.

6. Higashino R. Relationship between jaws and the masseter muscle by superimposing MR images on the cephalogram. The stomatological Society Journal 2006; 73:116-124.

7. Kubota M, Nakano H, Satoh K, Kamegai T, Ishikawa F et al. Human maxillofacial morphology related to masseter thickness, biting force and occlusal contact area. Dent J Iwate Med Univ 1998; 23:16-26.

8. Ishii C, Aragaki Y, Nagaki E, Honda R, Yotsui Y et al. Relationship between maxillofacial morphology and both volume and inclination of masseter muscle. Comparison between mandibular prognathism and normal subjects. Jpn J Jaw Deform 2004; 14:11-17.

9. Ogasawara K. Effects of the loss of occlusal supports on displacement of mandible and masticatory muscle activity. The Stomatological Society Journal 1986; 53:81-101.

10. Inaba K. A study on the relationship between the maxillofacial morphology and the biting force. The Shika Gakuho 1994; 94:153-175.

11. Takizawa T, Ohmori H, Moriya Y, Tsuchida K, Moriya Y et al. The influence of craniofacial form on bite force and EMG activity of masticatory muscles. VII-1. Correlative analysis of symmetry differences of frontal dentoskeletal form and mechanism of jaw function. Nihon Univ Dent $\mathbf{J}$ 1995; 69:904-915.
12. Uehara M, Fukuda S, Nakata S, and Nakasima A. A study on the relationship between occlusal pressure distribution and maxillofacial morphology in adult subjects with normal occlusion. Orthod Waves 2000; 59:98-110.

13. Inoue M, Nagata Y, Yotsui Y, Shimizutani K, and Kawamoto T. Relationship between bite force and maxillofacial morphology in normal occlusion. Jpn J Jaw Deform 2006; 16:176-183.

14. Carlson KE, Alston W, and Feldman DJ. Electromyographic study of agoing in skeletal muscle. Am J Phys Med 1964; 43:141-145.

15. Campbell MJ, McComas AJ, and Petitio F. Physiological change in aging muscles. J Neurol Neurosurg and Psychiatry $1973 ; 36: 174-182$.

16. Visser SL, and De Rijke W. Influence of sex and age on EMG contraction pattern. Eur Neurol 1974; 12:229-235.

17. Larsson L, Morphological and functional characteristics of the ageing skeletal muscle in man. Across sectional study. Acta Physiol Scand (suppl) 1978; 457:1-36.

18. Newton JP, and Yemm R. Changes in the contractile properties of the human first dorsal onterosseous muscle with age. Gerontol 1986; 32:98-104.

19. Newton JP, Yemm R, and McDonagh MJN. Study of age changes in the motor units of the first dosal interosseous muscle in man. Gerontol 1988; 34:115-119.

20. Von Karl Eichner. Über eine gruppeneinteilung der lückengebisse für die prothetik. Dez-Heft 1955; 1831-1834.

21. Ikebe K, Matsuda K, Murai S, Maeda Y, and Nokubi T. Validation of the Eichner index in relation to occlusal force and masticatory performance. The International Journal of Prosthodontics 2010; 23:521-524.

22. Miyachi T. A necessary condition and the sufficient condition of the index that you should measure by adult dental check up-from a deficit prosthetic view point-Gunji A and Shinjo F. A dentistry health strategy to 8020. Japan health promotion and fitness foundation. As for the making of health, it is a research trust business report 1989. 1990; 131-154.

23. King IT. The effect of water temperature on hand volumetric measurement using the water displacement method. J Hand Ther 1993; 6:202-204.

24. Kitahara M. Anatomical analysis of human masseter using MRI. Showa Igakukai Zzashi 1990; 50:507-512.

25. Watanabe A, Yamaga T, Ishioka K, Kiryu T and Saito Y. Electromyographic feature of complete denture wearers. Part 1. Comparison between complete denture wearers and the subjects with complete dentitions. J Jpn Prosthodont Soc 1987; 31:126-138.

26. Ihara K. Myofibrous organization of the m. masseter in man. Showa Igakukai Zzashi 1981; 41:519-526.

27. Yoshida R. Study on the differentiation and development of muscle fibers of masticatory muscles in mice in fed a liquid diet. J Jpn Orthod Soc 1995; 54:52-63.

28. Yamaguchi R, Yamazaki T, and Shiomoto S. Effect of stretch frequency per day in retarding disuse atrophy in rat soleus muscle. Ishikawa journal of physical therapy 2003; 3:3-7. 